\title{
- Mechanisms of Single-Electron Capture by the Dichlorocarbene Dication
}

\author{
Bernard Leyh* and Dimitri Hautot ${ }^{\dagger}$ \\ Département de Chimie, Université de Liège, Liège 1, Belgium
}

\begin{abstract}
The single-electron capture (SEC) by dichlorocarbene dications with eight different atomic and molecular target gases, $\mathrm{CCl}_{2}^{2+}+\mathrm{G} \rightarrow \mathrm{CCl}_{2}^{+}+\mathrm{G}^{+}$, has been studied by product ion spectroscopy and ion kinetic energy spectroscopy. The experimental data have been interpreted in the framework of a theoretical model that describes the charge exchange process. Exothermic charge exchange is handled within the Landau-Zener model, whereas endothermic charge exchange is described by the Demkov model. The calculated data reproduce qualitatively the essential features of the experimental results: (1) the appearance of a reaction window centered at an exothermicity in the 4-4.5-eV range, (2) the lower SEC cross sections for endothermic charge exchange, (3) the wider internal energy distributions obtained for $\mathrm{CCl}_{2}^{+}$in the endothermic regime than in the exothermic one, which results in larger dissociation yields, (4) the excitation of molecular targets that accompany their ionization in the SEC process, and (5) the kinetic energy released on the $\mathrm{CCl}^{+}+\mathrm{Cl}$ fragments in dissociative SEC. (J Am Soc Mass Spectrom 1996, 7, 266-275)
\end{abstract}

$\mathrm{T}$ The statistical theories of mass spectra play a central role in the rationalization of many mass spectral data because many molecular ions have been shown to dissociate according to the statistical hypotheses [1-3]. As a consequence, the rate constants for the competitive dissociation processes of a given molecular ion depend only on one variable parameter - the total internal energy - and are given by the well known Rice-Ramsperger-Kassel-Marcus quasi-equilibrium theory equation $[1,3,4]$. From this vantage point, one essential point in mass spectroscopic structural analysis is to be able to modulate the amount of internal energy deposited into the molecular ion of interest by the ionization or activation process. Tandem mass spectrometry has at its disposal a broad arsenal of activation techniques, the mechanisms of which are different enough to allow for a variety of internal energy distributions [5-8]. These collisional processes can be classified into two main groups, dependent on whether or not the charge of the projectile ion is affected. Collision-activated dissociation (CAD) belongs to the latter group, whereas charge reversal, charge stripping, single-electron capture, and so forth belong to the former. It need not be stressed that a good knowledge of the mechanisms involved in the activation step is a basic condition for an efficient use

Address reprint requests to Dr. Bernard Leyh, Departement de Chimie Générale et de Chimie Physique, Bâtiment B6c, Université de Liège, B. 4000 Sart-Tilman, Liège 1, Belgium.

* Chercheur qualifié du Fonds National de la Recherche Scientifique (Belgium).

${ }^{\dagger}$ Present affiliation: Department of Chemistry, University of Missouri-Rola, Rolla, MO 65401-0249. of the collisional process considered in a structural problem. Although their potentialities have been recognized [6], charge exchange processes in tandem mass spectrometry are not understood in depth, especially when molecular targets are concerned.

The present article deals with the internal energy distributions that result from single-electron capture (SEC) by fast (a few kiloelectronvolts) dications:

$$
\overrightarrow{\mathrm{M}}^{2+}+\mathrm{G} \rightarrow \overrightarrow{\mathrm{M}}^{+}+\mathrm{G}^{+}
$$

$\overrightarrow{\mathrm{M}}^{2+}$ is the fast projectile and $G$ is the collision gas (target gas).

Three aspects of this problem are of interest to us:

1. The equilibrium geometry of a doubly charged cation can be quite different from that of its singly charged counterpart. Therefore, single-electron capture can lead to a singly charged cation far away from its equilibrium geometry, which results in a quite specific dissociation dynamics.

2. The amount of internal energy can be modulated by change of the collision gas (target gas). For exothermic charge exchange, evidence has been found of a "reaction window," where the cross section is maximum [9-21]. However, endothermic charge exchange, for which energy has to be borrowed from the translational motion, leads to internally excited ions, which dissociate with large yields [21].

3. When molecular targets are used, one cannot neglect the possibility of target excitation (both vibrational and electronic) upon collisional ionization. This will, of course, influence the internal energy distribution of the projectile [21]. 


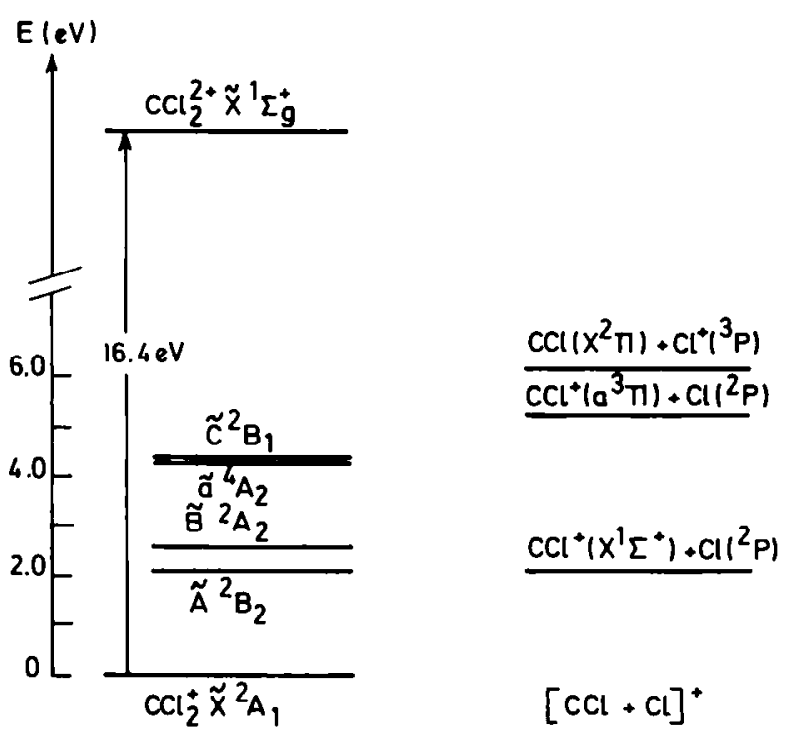

Figure 1. Thermochemistry of the $\mathrm{CCl}_{2}^{2+}$ and $\mathrm{CCl}_{2}^{+}$ions. The electronic state energies of $\mathrm{CCl}_{2}^{+}$have been taken from refs 29 and $28\left(\bar{a}^{4} A_{2}\right.$ state). The dissociation energies are from ref 34 .

In this work, we consider single-electron capture by the dichlorocarbene dication, which is a continuation of our previous study of the structure and reactivity of this dication [22]. The thermochemistry of $\mathrm{CCl}_{2}^{2+}$ already has been addressed by charge stripping experiments [23, 24] and by appearance energy measurements $[22,25,26]$. Data that concern the energetics of the ground state and the first excited states of $\mathrm{CCl}_{2}^{+}$ have been obtained by photoionization [27], charge reversal [28], and theoretical calculations [28, 29]. These data are summarized in Figure 1. At ion internal energies below $6 \mathrm{eV}$, the only open dissociation channel is $\mathrm{CCl}^{+}+\mathrm{Cl}$, on which we will focus our attention in the present article. We have used eight different target gases to address both the thermochemistry of the SEC process and the possible excitation of molecular targets. The experimental results are presented after a brief description of the experimental technique. A model for the charge exchange process then is introduced and its predictions are compared with the experimental data.

\section{Experimental}

A forward geometry double-focusing AEI-MS9 mass spectrometer upgraded with one collision cell in each field-free region has been used for all the experiments described in this article. Two kinds of spectra have been recorded: fragment ion spectra and ion kinetic energy spectra. Fragment ion spectra for decompositions that take place in the first field-free region have been measured by linked scan of the electrostatic analyzer and the magnet at a constant $B / E$ ratio [30]. Because the conversion of doubly charged ions into singly charged ions is under observation, the source accelerating voltage has to be set at half its normal value ( $4 \mathrm{kV}$ here). Decompositions that occur in the first field-free region also have been monitored by ion kinetic energy spectroscopy by using the accelerating voltage scan technique [31].

The experimental conditions were as follows: trap current, $30 \mu \mathrm{A}$; electron energy, $70 \mathrm{eV}$. In the linked scans at constant $B / E$, the resolution of the electrostatic analyzer was given by $\Delta E_{\mathrm{k}} / E_{\mathrm{k}}=2.5 \times 10^{-3}$. $\mathrm{CCl}_{2}^{2+}$ results from dissociative electron ionization of $\mathrm{CCl}_{4}$ (purity $=99.8 \%$ ). The target gases had the following purities: rare gases ( $>99.99 \%)$; nitrogen (99.8\%); benzene (99.7\%). The target gas pressure was low enough to ensure single-collision conditions (transmittance of the precursor beam $>90 \%$ ) $[32,33]$.

\section{Experimental Results}

\section{Separation of the Collision-Activated Dissociation and Single-Electron Capture Contributions}

When doubly charged ions collide with a neutral target, various processes can take place, which lead, among others, to singly charged parent and fragment ions. Two competitive mechanisms lead to fragment monocations, like $\mathrm{CCl}^{+}$in the present work:

$$
\mathrm{CCl}_{2}^{2+}+\mathrm{G} \rightarrow \mathrm{CCl}^{+}+\mathrm{Cl}+\mathrm{G}^{+} \text {(dissociative SEC) }
$$

$$
\mathrm{CCl}_{2}^{2+}+\mathrm{G} \rightarrow \mathrm{CCl}^{+}+\mathrm{Cl}^{+}+\mathrm{G} \quad(\mathrm{CAD})
$$

It is not possible to separate these two processes in a fragment ion spectrum obtained by linked scan at constant $B / E$. However, these can be separated in an ion kinetic energy spectrum because the amount of kinetic energy released on the fragments in the CAD process is much larger than in dissociative SEC, due to the repulsion between the singly charged fragments. In the CAD process described by eq 3 , for example, the minimum kinetic energy release is $\sim 3.5 \mathrm{eV}$ [22], whereas the average kinetic energy release for dissociative SEC (eq 2) is 1 order of magnitude smaller (vide infra, Table 1). As a consequence, the observed signal in the ion kinetic energy spectrum is the superposition of a dish-topped peak (CAD contribution) and of a Gaussianlike peak (dissociative SEC; see Figure 3 of ref 22).

To obtain "pure" CAD or dissociative SEC branching ratios for the different dissociation channels from the linked scan at constant $B / E$ spectra and from the accelerating voltage scan spectra, two problems have to be addressed:

1. The relative weight of the two contributions must be evaluated. Two different procedures that allow 
Table 1. Dissociation yield, SEC versus CAD ratio, and kinetic energy released on the $\mathrm{CCl}^{+}+\mathrm{Cl}$ fragments following single-electron capture by $\mathrm{CCl}_{2}^{2+}$ dications with different target gases

\begin{tabular}{lcccc}
\hline Target gas & $\begin{array}{c}\text { Target gas lowest } \\
\text { ionization energy }(\mathrm{eV})\end{array}$ & $\left(\frac{\mathrm{I}_{\mathrm{CCl}}^{+}}{\mathrm{I}_{\mathrm{CCI} I^{+}}}\right)_{\mathrm{SEC}}$ & $\frac{\mathrm{I}_{\mathrm{CCI}^{+}, \mathrm{SEC}}}{\mathrm{ICCl}^{+} \mathrm{CAD}}$ & $\begin{array}{c}\vec{\varepsilon}(\mathrm{eV}) \\
\left.(\mathrm{CCl})^{+}+\mathrm{Cl}\right)_{\mathrm{sEC}}\end{array}$ \\
\hline \hline $\mathrm{C}_{6} \mathrm{H}_{6}$ & 9.25 & $0.80 \pm 0.05$ & $28 \pm 3$ & $0.47 \pm 0.03$ \\
$\mathrm{CCl}_{4}$ & 11.47 & $0.06 \pm 0.01$ & $16 \pm 2$ & $0.31 \pm 0.03$ \\
$\mathrm{Xe}$ & 12.13 & $0.11 \pm 0.02$ & $6.7 \pm 0.7$ & $0.26 \pm 0.03$ \\
$\mathrm{Kr}$ & 14.00 & $0.20 \pm 0.02$ & $3.7 \pm 0.4$ & $0.34 \pm 0.03$ \\
$\mathrm{~N}_{2}$ & 15.58 & $0.36 \pm 0.02$ & $2.2 \pm 0.2$ & $0.44 \pm 0.06$ \\
$\mathrm{Ar}$ & 15.76 & $0.50 \pm 0.04$ & $2.1 \pm 0.2$ & $0.40 \pm 0.04$ \\
$\mathrm{Ne}$ & 21.56 & $1.86 \pm 0.10$ & $1.7 \pm 0.2$ & $0.35 \pm 0.03$ \\
$\mathrm{He}$ & 24.59 & $0.56 \pm 0.04$ & $1.3 \pm 0.3$ & $0.37 \pm 0.04$ \\
\hline
\end{tabular}

these two contributions to separate have been used and discussed in detail in our previous work [22], which was mostly concerned with the collisionactivated decomposition of the $\mathrm{CCl}_{2}^{2+}$ dication. It was observed inter alia, that the SEC/CAD intensity ratio increases when the target gas ionization energy decreases.

2. Corrections must be introduced to take into account the discrimination effect associated with the limited energy bandpass of the electrostatic analyzer in the $\mathrm{B} / \mathrm{E}$ linked scanning technique. In such spectra, fragment ions are discriminated, especially when they carry large kinetic energy releases. A procedure to correct for this effect has been discussed in refs 21 and 22.

In this article, we will concentrate on the production of $\mathrm{CCl}_{2}^{+}$and $\mathrm{CCl}^{+}$that results from SEC. As discussed in refs 21 and 22, the intensity ratio for $\mathrm{CCl}^{+}$that results from SEC only and is corrected for the abovementioned discrimination effect can be obtained via the following formula:

$$
=I_{\mathrm{CCl}+}^{\text {observed }} I_{\mathrm{CCl}^{+}, \mathrm{SEC}}^{\text {corrected }}+\mathrm{CAD} \frac{\int_{0}^{\infty} I_{\mathrm{SEC} V \text {-scan }}\left(E_{\mathrm{k}}\right) d E_{\mathrm{k}}}{\int_{\text {bandpass }} I_{\mathrm{SEC}+\mathrm{CAD}, v \text {-scan }}\left(E_{\mathrm{k}}\right) d E_{\mathrm{k}}}
$$

where $I_{C C I^{+}, S E C+C A D}^{\text {observed }}$ is the intensity measured in a linked scan spectrum at constant B/E, $I_{\mathrm{SEC}, \mathrm{V} \text {-scan }}\left(E_{k}\right)$ is the dissociative SEC contribution in the ion kinetic energy spectrum, and $I_{\mathrm{SEC}+\mathrm{CAD}, v \text {-scan }}\left(E_{\mathrm{k}}\right)$ is the total $(\mathrm{SEC}+\mathrm{CAD})$ intensity in the ion kinetic energy spectrum. The bandpass of the electrostatic analyzer in our linked scan spectra was given by $\Delta E_{\mathrm{k}} / E_{\mathrm{k}}=2.5 \times$ $10^{-3}$. This procedure, therefore, requires the measurement of product ion spectra and ion kinetic energy spectra at compatible acceleration voltages.

\section{Dissociation Yield to $\mathrm{CCl}^{+}+\mathrm{Cl}$}

Table 1 gives the $\left(I_{\mathrm{CCl}^{+}} / I_{\mathrm{CCl}_{2}^{+}}\right)_{\mathrm{SEC}}$ ratio obtained by using eq 4 for the different target gases. The SEC versus $C A D$ ratio also is recalled in an additional column. The variation of enthalpy for reaction 2 is given by eq 5 if ground state reactant and products are considered $[24,34,35]$ :

$$
\begin{aligned}
\Delta_{\mathrm{r}} H & =\mathrm{IE}(\mathrm{G})-\mathrm{IE}\left(\mathrm{CCl}_{2}^{+}\right)+\mathrm{D}\left(\mathrm{CCl}^{+}-\mathrm{Cl}\right) \\
& =\mathrm{IE}(\mathrm{G})-14.3 \mathrm{eV}
\end{aligned}
$$

where IE $(X)$ is the ionization energy of $X(X=G$ or $\left.\mathrm{CCl}_{2}^{+}\right)$and $\mathrm{D}\left(\mathrm{CCl}^{+}-\mathrm{Cl}\right)$ is the dissociation energy of $\mathrm{CCl}_{2}^{+}$to give $\mathrm{CCl}^{+}+\mathrm{Cl}$.

For exothermic dissociative SEC (target gases = $\mathrm{C}_{6} \mathrm{H}_{6}, \mathrm{CCl}_{4}$, and $\mathrm{Xe}$ ), these data show that a substantial amount of dissociation is observed only when benzene is the target gas. In this case, the exothermicity of the dissociative SEC reaction is equal to $5 \mathrm{eV}$. These data suggest that a given exothermicity has to be reached before the cross section starts to increase substantially.

For endothermic charge exchange, large fragmentation yields $\left(I_{\mathrm{CCl}^{+}} / I_{\mathrm{CCl}{ }_{2}^{2}}\right)_{\mathrm{SEC}}$ are observed, especially with $\mathrm{Ar}, \mathrm{Ne}$, and $\mathrm{He}$. These large dissociation probabilities coincide with a low global SEC cross section, as shown in the fourth column of Table 1. This also will be pointed out in the next subsection.

\section{Relative Single-Electron Capture Cross Sections for} the Production of $\mathrm{CCl}_{2}^{+}$and $\mathrm{CCl}^{+}$

Figure 2 shows the relative cross section for the production of $\mathrm{CCl}_{2}^{+}$and of $\mathrm{CCl}^{+}$as a function of the target gas. These data were recorded under identical ion source and transmittance conditions. As will be discussed in the next section, the thermochemical balance of the SEC reaction 1 is a central quantity in the present problem. The variation of enthalpy for this process when it involves ground state species is given by eq 6 [24]:

$$
\Delta_{\mathrm{r}} H=\mathrm{IE}(\mathrm{G})-\mathrm{IE}\left(\mathrm{CCl}_{2}^{+}\right)=\mathrm{IE}(\mathrm{G})-16.4 \mathrm{eV}
$$

Therefore, the experimental quantity we have chosen to label each target gas is its lowest ionization energy. 


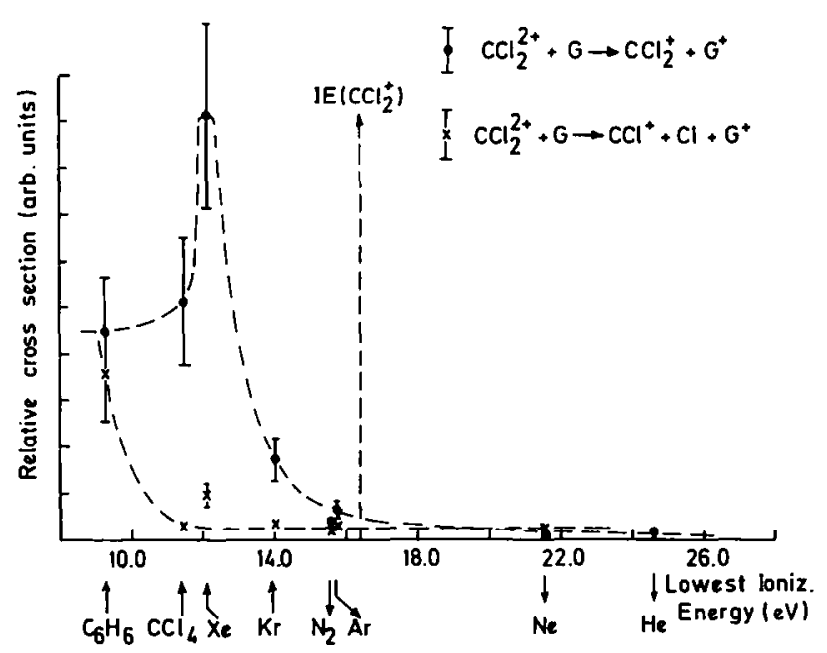

Figure 2. Experimental relative cross section for the nondissociative $\mathrm{SEC}$ process (dots) $\mathrm{CCl}_{2}^{2+}+\mathrm{G} \rightarrow \mathrm{CCl}_{2}^{+}+\mathrm{G}^{+}$and for the dissociative SEC process (crosses) $\mathrm{CCl}_{2}^{2+}+\mathrm{G} \rightarrow \mathrm{CCl}^{+}+\mathrm{Cl}+$ $\mathrm{G}^{+}$. The dotted lines are only an aid for the eye.

As far as $\mathrm{CCl}_{2}^{+}$is concerned, the data of Figure 2 show unambiguously that the single-electron capture cross section is maximum within a reaction window centered at a target gas ionization energy of about $12 \mathrm{eV}$. According to eq 6, this corresponds to an exothermicity of about $4.4 \mathrm{eV}$. Values in this range already have been found for a few molecular dications [14-21]. In the $\mathrm{NH}_{3}^{2+} \rightarrow \mathrm{NH}_{3}^{+}$case, for example, an optimum exothermicity of $4.5 \mathrm{eV}$ was deduced [21]. For the production of $\mathrm{CCl}^{+}$, Figure 2 substantiates the data of Table 1. According to eq 5 and with an optimal exothermicity of $4.4 \mathrm{eV}$, one should expect a maximum in the $\mathrm{CCl}^{+}$curve for target gases that have an ionization energy of $9.9 \mathrm{eV}$ : this is consistent with the rise observed at the ionization energy of $\mathrm{C}_{6} \mathrm{H}_{6}$.

The large dissociation yields mentioned in Table 1 for $\mathrm{Ar}, \mathrm{Ne}$, and Xe are obscured in Figure 2 due to the low global SEC cross section observed for these gases.

\section{Kinetic Energy Released on the $\mathrm{CCl}^{+}+\mathrm{Cl}$ Fragments}

The separation procedure between CAD and dissociative SEC described in refs 21 and 22 also allows estimation of the average kinetic energy released on the $\mathrm{CCl}^{+}+\mathrm{Cl}$ fragments that result from dissociative SEC. The kinetic energy release distributions (KERD) are obtained by differentiation of the SEC contribution [36, 37]; average kinetic energy releases are calculated from these KERD and are mentioned in the fifth column of Table 1 . The average kinetic energy releases also are plotted in Figure 3: a minimum is observed at a target ionization energy of $12 \mathrm{eV}$. As will be discussed in more detail in the next section, the average kinetic energy released on the fragments increases with increasing internal energy. It is therefore not surprising that a minimum in the $\bar{\varepsilon}$ versus $\operatorname{IE}(G)$ curve coincides

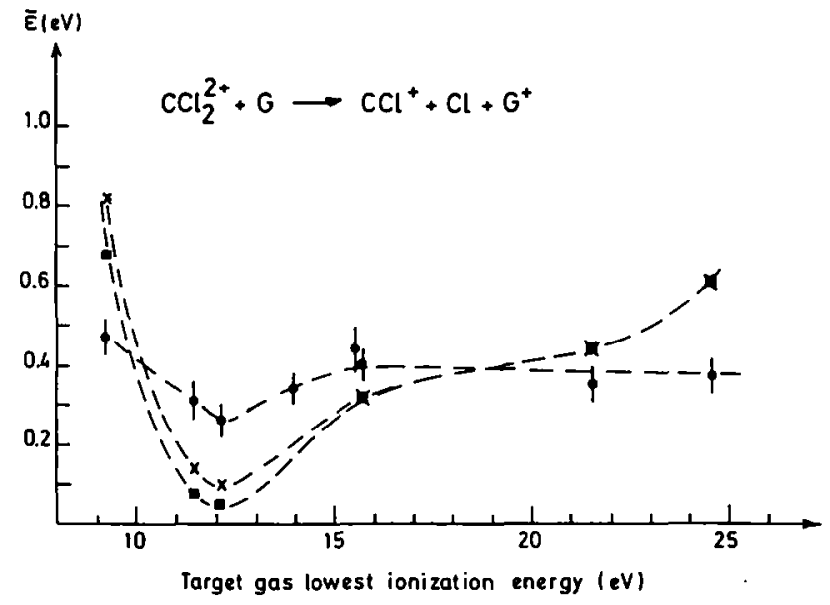

Figure 3. Average kinetic energy released on the $\mathrm{CCl}^{+}+\mathrm{Cl}$ fragments of the dissociative SEC reaction. Dots denote experiment, squares denote calculated with $E_{\text {int }}\left(\mathrm{CCl}_{2}^{2+}\right)=0 \mathrm{eV}$, and crosses denote calculated with $E_{\mathrm{int}}\left(\mathrm{CCl}_{2}^{2+}\right)=1 \mathrm{eV}$. The dotted lines are only an aid for the eye.

with the maximum of the $\mathrm{CCl}_{2}^{+}$cross section because both extrema are related to low internal energy $\mathrm{CCl}_{2}^{+}$ ions.

A model that describes the charge capture process should be able to account for the different features highlighted by the experimental results:

1. The appearance of a reaction window for exothermic charge exchange.

2. The change of behavior when one switches from the exothermic to the endothermic regime: lower total SEC cross section, but larger dissociation yield $\left(I_{\mathrm{CCl}^{+}} / I_{\mathrm{CCl}_{2}^{+}}\right)_{\mathrm{SEC}}$.

3. The dissociation dynamics of $\mathrm{CCl}_{2}^{+}$, that is, its dissociation yield and the associated kinetic energy release.

The next section is devoted to the description of such a model, whose performances will be evaluated based on the three criteria we have just mentioned.

\section{Theoretical Model}

\section{The Charge Exchange Process}

In its simplest form, the single-electron capture process can be described as a two-channel interaction that involves two potential energy surfaces of the supermolecule $\left[\mathrm{MG}^{++}\right]$[38]. The entrance channel corresponds to the $\mathrm{M}^{2+}+\mathrm{G}$ partners, whereas the exit channel corresponds to $\mathrm{M}^{+}+\mathrm{G}^{+}$. If the SEC process is exothermic, the diabatic potential energy surfaces associated with both channels will cross at a given $M-G$ distance, $R_{\mathrm{c}}$ (Figure $4 \mathrm{a}$ ). At this point the transition probability between both surfaces will be maximum. Such a case is expected to be satisfactorily described by the Landau-Zener model [38-40]. If, however, the SEC reaction is endothermic (Figure $4 b$ ), no crossing is 

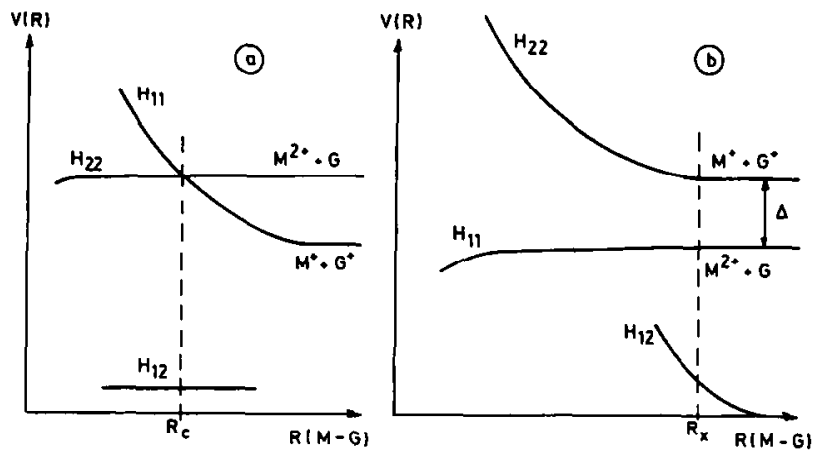

Figure 4. Schematic behavior of the long range diabatic potential energy surfaces along the target-projectile coordinate for (a) exothermic and (b) endothermic single-electron capture by dications. $\mathrm{H}_{12}$ is the coupling between the diabatic states. $\mathrm{H}_{11}$ and $\mathrm{H}_{22}$ are the diabatic energies. In the Landau-Zener model (a), $\mathrm{H}_{12}$ is assumed to be constant in the coupling zone, whereas it varies exponentially in the Demkov model (b).

expected and a more appropriate model is the Demkov model $[38,41]$.

Bimolecular charge exchange involves both the formation of the $\left[\mathrm{MG}^{2+}\right]$ transient and its further dissociation, in other words, a double passage in the coupling zone. The lifetime of the collision complex is very short (a few femtoseconds when the translational energy is in the kiloelectronvolt range) and in any case much shorter than an intramolecular vibration of either $\mathrm{M}$ or G. If $p_{1}$ is the probability of remaining on the same diabatic surface, the SEC probability for a double crossing of the coupling zone is given by

$$
p_{\mathrm{SEC}}=2 p_{1}\left(1-p_{1}\right)
$$

Depending on the exothermic or endothermic nature of the reaction, we will choose either the Landau-Zener or the Demkov approximation for the transition probability. In the Landau-Zener model [38-40], the cross sections in the diabatic surfaces along the $\mathrm{M}-\mathrm{G}$ coordinate (target-projectile distance) are supposed to be linear in the vicinity of the crossing point $R_{\mathrm{c}}$ and the interaction between the surfaces, $\mathrm{H}_{12}$, is assumed to remain constant in this domain. The probability $p_{1}$ is then given by

$$
p_{\mathrm{LZ}}(b)=\exp \left\{-\frac{2 \pi H_{12}^{2}}{\hbar v_{0}|\Delta F|}\left[1-\left(\frac{b}{R_{c}}\right)^{2}\right]^{-1 / 2}\right\}
$$

where $b$ is the impact parameter, $v_{0}$ is the relative target-projectile velocity, and $|\Delta F|$ is the slope difference of the diabatic surfaces at the crossing point.

In the Demkov model [41] (also called the Rosen-Zener model), the diabatic surfaces are supposed to be parallel and separated by an energy increment $\Delta$ in the coupling zone. In this region, an exponential form is assumed for the coupling element,

$$
\mathrm{H}_{12}(R)=A \exp (-\alpha R)
$$

and the probability to remain on a diabatic surface is equal to

$$
p_{\mathrm{D}}(b)=\left\{1+\exp \left\{\frac{\pi \Delta}{\hbar \alpha v_{0}}\left[1-\left(\frac{b}{R_{x}}\right)^{2}\right]^{-1 / 2}\right\}\right\}^{-1}
$$

The target-projectile distance $R_{x}$ is given by

$$
R_{x}=\frac{1}{\alpha} \ln \frac{2 A}{\Delta}
$$

The single-electron capture cross section is then obtained by integration over all possible values of the impact parameter:

$$
\sigma_{\mathrm{SEC}}=2 \pi \int_{0}^{\infty} P_{\mathrm{SEC}}(b) b d b
$$

Trajectories with $b$ values larger than $R_{c}$ (LandauZener model) or $R_{x}$ (Demkov model) are assumed not to contribute to the SEC cross section.

\section{Application to the $\mathrm{CCl}_{2}^{2+} \stackrel{\mathrm{G}}{\rightarrow} \mathrm{CCl}_{2}^{+}$Single-Electron Capture}

The coupling element between the diabatic surfaces has been calculated according to the empirical formula of Kimura et al. [11] (eqs 13 and 15). This formula has the advantage that it is of exponential type and thus directly applicable in the Demkov model.

For the Demkov model, the following formulas have been used in addition to the abovementioned ones:

$$
\mathrm{H}_{12}(R)=3.875 \exp [-(1.324 \sqrt{\mathrm{IE}(G)}) R]
$$

$\Delta=$ asymptotic energy difference between entrance and exit channel

$$
\begin{aligned}
= & \mathrm{IE}_{0}(\mathrm{G})-\mathrm{IE}_{0}\left(\mathrm{CCl}_{2}^{+}\right)+E_{\mathrm{int}}\left(\mathrm{G}^{+}\right) \\
& -E_{\mathrm{int}}\left(\mathrm{CCl}_{2}^{2+}\right)+E_{\mathrm{int}}\left(\mathrm{CCl}_{2}^{+}\right)
\end{aligned}
$$

where $\mathrm{IE}_{0}$ is the lowest ionization energy and $E_{\text {int }}$ is the internal energy for the species mentioned in parentheses.

For the Landau-Zener model, we used the equations

$$
\begin{gathered}
\mathrm{H}_{12}=3.875 \exp \left(-1.324 R_{c} \sqrt{\mathrm{IE}(G)}\right) \\
R_{c}=\frac{1}{|\Delta|} \\
|\Delta F|=\frac{1}{R_{c}^{2}}
\end{gathered}
$$

$\Delta$ has the same meaning for both models. Equations 13-17 are given in atomic units. 
The target gases $\mathrm{C}_{6} \mathrm{H}_{6}, \mathrm{CCl}_{4}$, and $\mathrm{Xe}$ all give rise to exothermic charge exchange $(\Delta<0)$ in the $\mathrm{CCl}_{2}^{+}$internal energy domain investigated and can therefore be described by the Landau-Zener model. $\mathrm{Ne}$ and $\mathrm{He}$ lead to endothermic SEC $(\Delta>0)$ and are therefore described by the Demkov model. $\mathrm{Kr}, \mathrm{Ar}$, and $\mathrm{N}_{2}$ are intermediate. The dissociation energy of $\mathrm{CCl}_{2}^{+}$to give $\mathrm{CCl}^{+}+\mathrm{Cl}$ is equal to $2.1 \mathrm{eV}$ [34]. According to eq 5, dissociative SEC with $\mathrm{Kr}$ will be slightly exothermic. On the other hand, nondissociative SEC with $\mathrm{Ar}$ and $\mathrm{N}_{2}$ also will be slightly exothermic. Such threshold situations are not described satisfactorily by either the Landau-Zener or the Demkov model. However, nondissociative SEC with $\mathrm{Kr}$ is exothermic enough to be handled by the Landau-Zener approximation, and dissociative SEC with Ar is endothermic enough to be handled by the Demkov model.

Electronic excitation of the ionized target also must be considered. For the rare gases $\mathrm{Ar}, \mathrm{Kr}$, and $\mathrm{Xe}$, the two electronic states that can be accessed, ${ }^{2} P_{3 / 2}$ and ${ }^{2} P_{1 / 2}$, have respective weights of 4 and 2 , equal to their degeneracies. For the $\mathrm{CCl}_{4}$ and $\mathrm{C}_{6} \mathrm{H}_{6}$ target gases, the accessible electronic states are known from photoelectron spectroscopy $[42,43]$. The calculations that will be discussed now have been performed for each electronic state with its own ionization energy, and the results have been averaged by using weights equal to their relative band intensities in the photoelectron spectrum. From the point of view of the target gas, this procedure assumes that SEC by fast dications, owing to the very short interaction time, consists of a nearly vertical ionization process like direct photoionization. Comparison with the experimental data allows us to check the validity of this procedure.

According to the foregoing models, we have calculated the cross section for the production of $\mathrm{CCl}_{2}^{+}$as a function of its internal energy. These calculations were performed for two values of the internal energy content of the $\mathrm{CCl}_{2}^{2+}$ dication: 0 and $1 \mathrm{eV}$. The two lowest dissociation asymptotes of $\mathrm{CCl}_{2}^{+}$are located at internal energies of $2.1 \mathrm{eV}\left(\mathrm{CCl}^{+}+\mathrm{Cl}\right)$ and $6.15 \mathrm{eV}\left(\mathrm{CCl}+\mathrm{Cl}^{+}\right)$ (Figure 1). From the calculated internal energy distributions, the cross sections for production of $\mathrm{CCl}_{2}^{+}$and $\mathrm{CCl}^{+}$were deduced by integration over their respective internal energy domain. These data are displayed in Figure 5 and agree qualitatively with the experimental data of Figure 2.

Three points deserve emphasis. First of all, in the exothermic regime, a maximum clearly is observed in the $\mathrm{CCl}_{2}^{+}$cross section at a target ionization energy of about $12 \mathrm{eV}$. The rise in the $\mathrm{CCl}^{+}$cross section with benzene as the target gas also is well reproduced. The Landau-Zener model accounts very well for the peak of the reaction window at an exothermicity of 4-4.5 $\mathrm{eV}$. According to eq 16, this exothermicity corresponds to an optimal $R_{c}$ value equal to $3.2-3.6 \AA$, which is in keeping with other results on single-electron capture $[10,14-21]$, that found that the reaction window spans the $2-8-\AA$ range for the charge-transfer distance $R_{c}$.

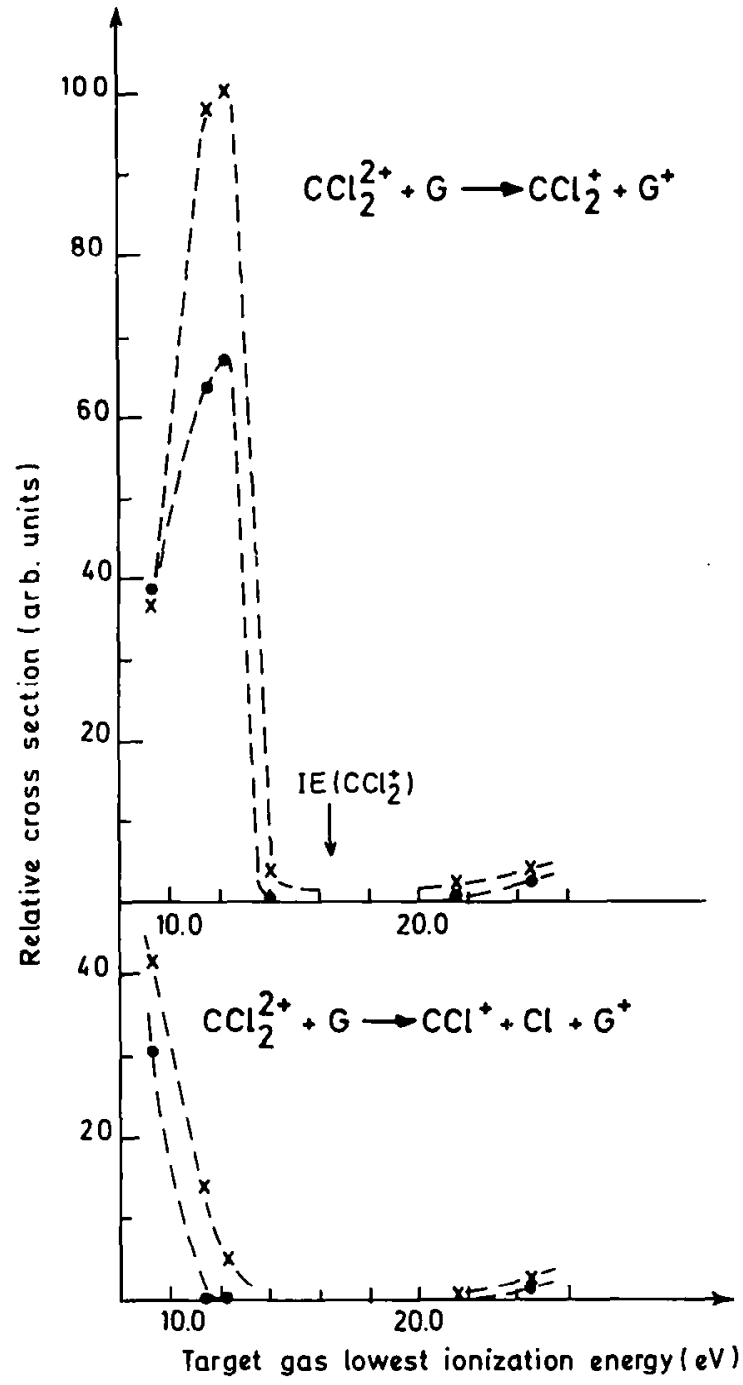

Figure 5. Calculated relative cross section for the nondissociative SEC process $\mathrm{CCl}_{2}^{2+}+\mathrm{G} \rightarrow \mathrm{CCl}_{2}^{+}+\mathrm{G}^{+}$(top) and for the dissociative $\mathrm{SEC}$ process $\mathrm{CCl}_{2}^{2+}+\mathrm{G} \rightarrow \mathrm{CCl}^{+}+\mathrm{Cl}+\mathrm{G}^{+}$(bottom). Dots denote calculations with $E_{\text {int }}\left(\mathrm{CCl}_{2}^{2+}\right)=0 \mathrm{eV}$ and crosses denote calculations with $E_{\text {int }}\left(\mathrm{CCl}_{2}^{2+}\right)=1 \mathrm{eV}$. The dotted lines are only an aid for the eye.

Second, the data summarized in Table 2 show also that the calculated average internal energies deposited in $\mathrm{CCl}_{2}^{+}$upon SEC and the theoretical dissociation yields qualitatively reproduce the experimental tendency toward an increase in internal energy while switching to the endothermic regime. A third observation is that the calculated data obtained for SEC by cold ( $E_{\text {int }}=0 \mathrm{eV}$ ) $\mathrm{CCl}_{2}^{2+}$ dications display a too abrupt variation as a function of the target gas. In other words, the experimental reaction window has smoother boundaries than the calculated window. This discrepancy is less dramatic when vibrationally hotter $\mathrm{CCl}_{2}^{2+}$ ions are considered (compare the data at $E_{\text {int }}=0$ and $1 \mathrm{eV}$ ). Increasing the $\mathrm{CCl}_{2}^{2+}$ internal energy is equivalent to moving the reaction window toward higher target gas ionization energies. The lowest dissociation barrier of $\mathrm{CCl}_{2}^{2+}$ is equal to $4.35 \mathrm{eV}$ [22] so that the dications that reach 
Table 2. Theoretical results obtained using the Demkov (D) or the Landau-Zener (LZ) model: average internal energy, dissociation yield, average internal energy of $\mathrm{CCl}_{2}^{+}$ions that dissociate in the $\mathrm{CCl}^{+}+\mathrm{Cl}$ channel, and average kinetic energy release on the $\mathrm{CCl}^{+}+\mathrm{Cl}_{\text {fragments }}$ using Klots' equation ${ }^{a}$

\begin{tabular}{lccccc}
\hline $\begin{array}{c}\text { Target } \\
\text { gas }\end{array}$ & Model & $\bar{E}_{\text {int }}(\mathrm{eV})$ & $\left(\frac{\mathrm{I}_{\mathrm{CCl}}^{+}}{\mathrm{ICCl}_{2}^{+}}\right)_{\mathrm{SEC}}$ & $\begin{array}{c}\bar{E}_{\text {int }}(\mathrm{eV}) \\
\mathrm{CCl}_{2}^{+} \rightarrow \mathrm{CCl}^{+}+\mathrm{Cl} \\
\left(2.1 \mathrm{eV}<E_{\mathrm{int}}<6.1 \mathrm{eV}\right)\end{array}$ & $\begin{array}{c}\bar{\varepsilon}(\mathrm{eV}) \\
\left.(\mathrm{CCl})^{+}+\mathrm{Cl}\right)_{\mathrm{SEC}}\end{array}$ \\
\hline \hline $\mathrm{C}_{6} \mathrm{H}_{6}$ & $\mathrm{LZ}$ & $2.10(2.81)$ & $0.79(1.14)$ & $3.72(4.11)$ & $0.68(0.83)$ \\
$\mathrm{CCl}_{4}$ & $\mathrm{LZ}$ & $0.73(1.21)$ & $3.6 \times 10^{-3}(0.15)$ & $2.24(2.41)$ & $0.07(0.14)$ \\
$\mathrm{Xe}$ & $\mathrm{LZ}$ & $0.53(1.18)$ & $1.5 \times 10^{-4}(0.05)$ & $2.20(2.31)$ & $0.05(0.10)$ \\
$\mathrm{Ar}$ & $\mathrm{D}$ & & & $2.83(2.83)$ & $0.31(0.31)$ \\
$\mathrm{Ne}$ & $\mathrm{D}$ & $1.16(1.14)$ & $0.19(0.18)$ & $3.14(3.13)$ & $0.44(0.44)$ \\
$\mathrm{He}$ & $\mathrm{D}$ & $2.40(2.39)$ & $0.57(0.57)$ & $3.57(3.56)$ & $0.61(0.61)$ \\
\hline
\end{tabular}

${ }^{a}$ The unbracketed values correspond to $E_{\text {int }}\left(\mathrm{CCl}_{2}^{2+}\right)=0 \mathrm{eV}$; the bracketed values correspond to $E_{\text {ini }}\left(\mathrm{CCl}_{2}^{2+}\right)=1 \mathrm{eV}$.

the collision cell after a few microseconds have an internal energy content between 0 and $4.35 \mathrm{eV}$. Although the exact form of this internal energy distribution is unknown, it can be anticipated from the data obtained at 0 and $1 \mathrm{eV}$ that an average over this distribution will make the cross section versus target gas curve smoother and thus closer to the experimental behavior. Let us also mention that this effect is particularly crucial in the exothermic regime, but much less sensitive in the endothermic regime.

Notwithstanding the qualitative agreement between calculated and experimental observables, it must be stressed that the crudeness of the model used implies that a more quantitative agreement reasonably could not be expected. In particular the use of the semiempirical equations 13 and 15 for the $\mathrm{H}_{12}$ coupling element is questionable and should be investigated more carefully. The large dissociation yield observed with Ne is not accounted for and we cannot explain precisely why.

\section{Kinetic Energy Release on the $\mathrm{CCl}^{+}+\mathrm{Cl}$ Fragments}

For dissociations that obey the hypotheses of statistical theories, the kinetic energy release distributions depend only on the internal energy above the dissociation asymptote, $E^{\ddagger}$, and on the fragment densities of states. The average kinetic energy released on the fragments, $\bar{\varepsilon}$ can be calculated from the theoretical internal energy distributions via Klots' equation [44-46]:

$$
E^{\ddagger}=\bar{\varepsilon}+\frac{r-1}{2} \bar{\varepsilon}+\sum_{i=1}^{s} \frac{h \nu_{i}}{\exp \left(h \nu_{i} / \bar{\varepsilon}\right)-1}
$$

where $r$ is the total number of rotational degrees of freedom of both fragments, $s$ is the number of vibrational degrees of freedom of the fragments, and $\nu_{i}$ are the vibrational frequencies in the harmonic approximation.
The calculated average kinetic energy releases $\bar{\varepsilon}$ are mentioned in the last column of Table 2. In Figure 3, they are compared to the experimental results. Once again, a qualitative agreement is observed, but the variation of the theoretical data is too pronounced. This effect decreases, however, as expected (vide supra), when $E_{\text {int }}\left(\mathrm{CCl}_{2}^{2+}\right)$ increases, so that the data could be improved by averaging over the (unknown) internal energy distribution of the starting dications. Particularly interesting is the minimum observed at $12-\mathrm{eV}$ target ionization energy. This can be correlated with the internal energies mentioned in Table 2. Low internal energies lead to low fragmentation yields and high parent ion intensity (see Table 1 and Figure 2) and also to low kinetic energy releases (see eq 18). High fragmentation yields and large kinetic energy releases can be obtained in two ways: in the exothermic regime with low ionization energy targets $\left(\mathrm{C}_{6} \mathrm{H}_{6}\right)$ and in the endothermic regime.

\section{Effects of the Vibrational Motion}

The model calculations presented up to now do not take the vibrational motion into account. This amounts basically to neglect of the interference between the exit channels. A complete treatment should:

1. Consider that the coupling elements $\mathrm{H}_{12}$ are in fact the product of an electronic factor given by eqs 13 and 15 and an overlap integral between the vibrational functions of both diabatic electronic states, in the interaction region, that is, at a target-projectile distance close to $R_{\mathrm{c}}$ or $R_{\mathrm{x}}$ [47].

2. Handle the problem as a multichannel problem.

Such a treatment is made very difficult owing to the many unknowns of the problem. The overlap integrals between the vibrational functions depend on the equilibrium geometries and force constants of both states, perturbed by the approach of the target. These data are unknown for the electronically excited states of $\mathrm{CCl}_{2}^{+}$. Furthermore, no practical method exists, to our knowl- 
edge, to take the interference effect into account. However, neglect of the interference effect is probably not only convenient, but also realistic. To quote Nakamura [38], "The favorable situations, however, might fortunately hold in many cases. ... When the colliding particles are heavy, the various phases accumulate easily and the random phase approximation might hold well." The purpose of the present article is not to present a sophisticated model to account for all aspects of the experimental data, but, rather, to explain the gross features of the SEC process and of the resulting dissociation dynamics of $\mathrm{CCl}_{2}^{+}$. The question we would like to answer in this subsection is the following: Are the $\mathrm{CCl}_{2}^{+}$ions that result from SEC initially vibrationally or electronically excited? In other words, does SEC lead to vibrationally hot ground state ions or to vibrationally cold but electronically excited ions?

To answer this question, we have considered the charge transfer process at high translational energy $(8 \mathrm{keV})$ as vertical and we have neglected the perturbation of the vibrational wave functions due to the approach of the target. In that case, the probability of production of a given vibrational state of $\mathrm{CCl}_{2}^{+}$is proportional to the Franck-Condon factor between the initial $\mathrm{CCl}_{2}^{2+}$ vibrational wave function and the final $\mathrm{CCl}_{2}^{+}$wave function. These Franck-Condon factors were calculated by using the recurrence formula of Manneback [48] in the harmonic approximation. The vibrational frequencies were calculated at the modified neglect of differential overlap level. The equilibrium geometries already were obtained in previous works $[22,28]$. Comparison of the equilibrium geometries (see legend of Table 3 ) shows that only the valence angle is substantially modified, whereas the dication is linear and the monocation is bent and thus characterized by a double-minimum potential along the valence angle coordinate. The overlap integrals calculated between the vibrationless state of $\mathrm{CCl}_{2}^{2+}$ and the vibrational states of $\mathrm{CCl}_{2}^{+}$excited in the $\nu_{1}$ mode are given in Table 3. For the $\nu_{2}$ mode, the situation is more complicated due to the double-minimum potential. According to Herzberg [49], two limiting cases exist. Below the inversion barrier $\left(1200 \mathrm{~cm}^{-1}\right.$ in this case),

Table 3. Franck-Condon factors between the vibrationless wave function $|0\rangle$ of $\mathrm{CCl}_{2}^{2+} \tilde{X}^{1} \Sigma_{\mathrm{g}}^{+}$and different vibrational wave functions $\left|v_{i}\right\rangle$ of $\mathrm{CCl}_{2}^{+} \tilde{X}^{2} A_{1}^{\text {a" }}$

\begin{tabular}{lccc}
\hline$v_{1}$ & $\left.\left\langle v_{1} \mid 0\right\rangle\right|^{2}$ & $v_{2}$ & $\left|\left\langle v_{2} \mid 0\right\rangle\right|^{2}$ \\
\hline \hline 0 & 0.843 & 0 & 0.037 \\
1 & 0.144 & 1 & 0.123 \\
2 & 0.012 & 2 & 0.202 \\
3 & $710^{-4}$ & 3 & 0.221 \\
4 & $310^{-5}$ & 4 & 0.182 \\
\hline
\end{tabular}

\footnotetext{
- The equilibrium geometries are the following. $\mathrm{CCl}_{2}^{2+} \bar{x}^{1} \Sigma_{\mathrm{g}}^{+}$: linear, $R_{\mathrm{C}-\mathrm{Cl}}=1.57 \AA$ : $\mathrm{CCl}_{2}^{+} \bar{x}^{2} A_{1}$ : bent, $R_{\mathrm{C}-\mathrm{Cl}}=1.59 \AA \AA^{2}, \Theta_{\mathrm{Cl}-\mathrm{C}-\mathrm{Cl}}=$ $160.5^{\circ}$. The vibrational frequencies are as follows. $\mathrm{CCl}_{2}^{2+} \bar{x}^{-} \Sigma_{0}^{+}$: $\nu_{1}=646 \mathrm{~cm}^{-1}, \nu_{2}=405 \mathrm{~cm}^{-1}$ (doubly degenerate), $\nu_{3}=1615$ $\mathrm{cm}^{-1} ; \mathrm{CCl}_{2}^{+} \bar{X}^{2} A_{1}: \nu_{1}=650 \mathrm{~cm}^{-1}, \nu_{2}=300 \mathrm{~cm}^{-1}, \nu_{3}=1510 \mathrm{~cm}^{-1}$.
}

we have two independent potentials with the same frequency. Each level is doubly degenerate, but the splitting is negligible at low energies. For energies larger than twice the barrier, the potential can be visualized as a single-minimum potential with half the vibration frequency. Our calculations show that the overlap integrals vanish for energies larger than the barrier. The results below the barrier are shown in Table 3.

Table 3 shows clearly that high vibrational states of the ground electronic state of $\mathrm{CCl}_{2}^{+}$will not be populated directly. The SEC process most probably will lead to excited electronic states (see Figure 1). We have shown in a previous work [28] that the density of electronic states is important in the $2-3-\mathrm{eV}$ internal energy range and that these states are connected with each other and with the ground state by very efficient nonadiabatic interactions (conical intersections, Renner-Teller coupling), which should make the quantum yield for internal conversion to the ground state close to unity $[50,51]$. To summarize, we suggest that when the target gas is appropriate to create a sufficiently excited $\mathrm{CCl}_{2}^{+}$ion, the initial electronic excitation is rapidly converted into vibrational energy of the ground state from which dissociation occurs.

\section{Conclusions}

The theoretical model based on the Landau-Zener and Demkov approximations for the charge exchange probability accounts qualitatively for the major features of the experimental data.

Depending on the ionization energy of the target gas, the single-electron capture process can be exothermic or endothermic. A first important observation is the appearance of a reaction window in the exothermic regime. This reaction window is centered at an exothermicity of $4-4.5 \mathrm{eV}$ and has a width at halfheight of $2 \mathrm{eV}$. The origin of this reaction window lies in eq 7. The SEC probability is maximum when the probability of remaining on the same diabatic potential energy surface, $p_{1}$, is equal to 0.5 . Examination of eqs 8 and 15-17 shows that this value will be reached for a given value of the exothermicity $\Delta$.

A consequence of this reaction window is that the internal energy distribution of the monocation that results from SEC will be relatively narrow $(\sim 2 \mathrm{eV}$ wide), at least if atomic targets are used. This is illustrated in Figure 6 in the case of Xe. If molecular targets are used, however, the existence of low-lying and closely spaced electronic states of the ionized target results in the possibility of exciting the target upon SEC. We have assumed in our calculations that the distribution of internal energy of $\mathrm{G}^{+}$obtained in this way is equivalent to that obtained by photoelectron spectroscopy. The comparison between theory and experiment (Figures 2, 3, and 5) is satisfactory enough to suggest that the actual state distribution is probably not too different from our assumption. As already 
observed for SEC by $\mathrm{NH}_{3}^{2+}$, electronic excitation of molecular targets, therefore, may not be neglected. Low ionization energy molecular targets actually can lead to less excited monocations $\left(\mathrm{CCl}_{2}^{+}\right.$here) because the energy available has been transferred partly to the ionized target.

The endothermic regime displays quite different behavior. Equations 7 and 10 show that in the Demkov model, $p_{\mathrm{SEC}}$ is maximum for resonant charge exchange $(\Delta=0)$. This is the situation observed, for charge exchange processes that involve slowly varying longrange potentials, like neutralization of monocations [52]. This resonance condition is not realized in our case with target gases like $\mathrm{Ne}$ or $\mathrm{He}$, which explains the low SEC cross section observed with these targets. However, these cross sections, though much smaller, also vary much more smoothly with energy so that the internal energy distribution of the $\mathrm{CCl}_{2}^{+}$ions will be much wider than in the exothermic case. This is clearly demonstrated in Figure 6, where the $\mathrm{Xe}$ and He cases are compared. As a consequence, the average internal energies and dissociation yields, $\left(I_{\mathrm{CCl}^{+}} / I_{\mathrm{CCl}_{2}^{+}}\right)_{\mathrm{SEC}}$, are larger with $\mathrm{He}$ and $\mathrm{Ne}$ than with $\mathrm{Xe}$ or $\mathrm{CCl}_{4}$, for example, in good agreement with the experimental data (see Table 1).

The internal energy variations brought about by using various target gases manifest themselves not only in the cross sections and dissociation yields, but also in the kinetic energy released on the dissociation fragments that result from dissociative SEC. Figure 3 shows that the reaction window appears here also as a $\sim 2-3-\mathrm{eV}$-wide minimum. This general tendency is accounted for by theory, although the calculated variation is too sharp. It is not possible to decide whether this discrepancy comes from the crudeness of the model used for charge exchange or from the use of Klots' equation to convert internal energies into kinetic energy releases. Klots' equation assumes a statistical redistribution of the internal energy, which is probably not fully realized in a triatomic ion. Let us also note

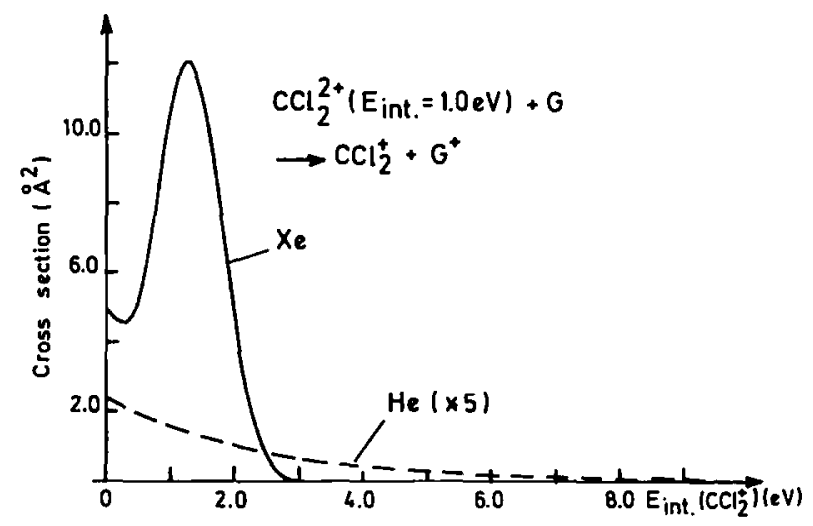

Figure 6. Calculated internal energy distributions of $\mathrm{CCl}_{2}^{+}$for exothermic SEC (target gas $=\mathrm{Xe}$ ) and endothermic SEC (target gas $=\mathrm{He}) . E_{\mathrm{int}}\left(\mathrm{CCl}_{2}^{2+}\right)=1 \mathrm{eV}$. For SEC with $\mathrm{Xe}$, the data shown are the weighted mean over the $\mathrm{Xe}^{+}{ }^{2} P_{3 / 2}$ and ${ }^{2} P_{1 / 2}$ states (see text). that it is possible to make the theoretical curves for both the cross sections and the kinetic energy release less sharp by taking into account the necessarily present vibrational energy content of the $\mathrm{CCl}_{2}^{2+}$ dication we start with. An increase of this energy content leads to a slight displacement of the reaction window toward higher energies: averaging over a distribution of internal energies of $\mathrm{CCl}_{2}^{2+}$ will result in smoother boundaries of the reaction window. This can explain why the experimental window is wider than the calculated window.

To summarize, switching from one target gas to another one has profound consequences in singleelectron capture experiments with dications. If SEC is exothermic, there exists a reaction window within which the SEC cross section is maximum. This window governs the internal energy distribution of the resulting monocation projectile. The internal energy distribution is relatively narrow $(\sim 2 \mathrm{eV})$, which allows either the undissociated parent ion or given dissociation channels to be favored. Endothermic SEC is much less intense and leads to much wider internal energy distributions and thus to much less selective dissociation channels.

\section{Acknowledgments}

We are grateful to Professor Robert Locht for a critical reading of the manuscript. Generous financial support from the Belgian Fonds de la Recherche Fondamentale Collective (Grants 2.4537.91F and 2.4532.95), from the Loterie Nationale (Grant 9.4610.90), and from the University of Liège is gratefully acknowledged.

\section{References}

1. Lorquet, J. C. Mass Spectrom. Rev. 1994, 13, 233-257.

2. Lifshitz, C. Int. J. Mass Spectrom. Ion Processes 1992, 118/119, 315-337.

3. Baer, T. Adv. Chem. Phys. 1986, 64, 111-202.

4. Forst, W. Theory of Unimolecular Renctions; Academic Press: New York, 1973.

5. McLafferty, F. W., Ed. Tandem Mass Spectrometry; Wiley-Interscience: New York, 1983.

6. Busch, K. L.; Glish, G. L.; McLuckey, S. A. Mass Spectrometry / Mass Spectrometry; VCH: Weinheim, 1988.

7. Cooks, R. G., Ed. Collision Spectroscopy; Plenum Press: New York, 1978.

8. Cooks, R. G.; Ast, T.; Kralj, B.; Kramer, V.; Zigon, D. J. Am. Soc. Mass Spectrom. 1990, 1, 16-27.

9. Olson, R. E.; Salop, A. Phys. Rev. A 1976, 14, 579-585.

10. Smith, D.; Adams, N. G.; Alge, E; Villinger, H.; Lindinger, W. J. Phys. B 1980, 13, 2787-2799.

11. Kimura, M.; Iwai, T.; Kaneko, Y.; Kobayashi, N.; Matsumoto, A.; Ohtari, S.; Okuno, K.; Takagi, S.; Tawara, H.; Tsurubushi, S. J. Phys. Soc. Jpn. 1984, 53, 2224-2232.

12. Taulbjerg, K. J. Phys. B 1986, 19, L367-L372.

13. Lee, A. R.; Wilkins, A. C. R.; Enos, C. S.; Brenton, A. G. Int. J. Mass Spectrom. Ion Processes 1994, 130, 83-88.

14. Koslowski, H. R.; Lebius, H.; Staemmler, V.; Fink, R.; Wiesemann, K.; Huber, B. A. J. Phys. B 1991, 24, 5023-5034.

15. Herman, Z.; Jonathan, P.; Brenton, A. G.; Beynon, J. H. Chem. Phys. Lett. 1987, 141, 433-442. 
16. Rogers, S. A.; Price, S. D.; Leone, S. R. J. Chem. Phys. 1993, 98, 280-289.

17. Reid, C. J.; Ballantine, J. A.; Harris, F. M. Int. J. Mass Spectrom. Ion Processes 1989, 93, 23-47.

18. Price, S. D.; Rogers, S. A.; Leone, S. R. J. Chem. Phys. 1993, 98, 9455-9465.

19. Mathur, D.; Reid, C. J.; Harris, F. M. J. Phys. B 1987, 20, L577-L581.

20. Manning, M.; Price, S. D.; Leone, S. R. J. Chem. Phys. 1993, 99, 8695-8704.

21. Leyh, B.; Hoxha, A. Chem. Phys. 1995, 192, 65-77.

22. Leyh. B.; Hautot, D. J. Am. Soc. Mass Spectrom. 1995, 6, 1019-1029.

23. Proctor, C. J.; Porter, C. J.; Ast, T.; Beynon, J. H. Int. J. Mass Spectrom. Ion Phys. 1982, 41, 251-263.

24. Langford, M. L.; Hamdam, M.; Harris, F. M. Int. J. Mass Spectrom. Ion Processes 1990, 95, 243-258.

25. Leiter, K.; Stephan, K.; Märk, E.; Märk, T. D. Plasma Chem. Plasma Proc. 1984, 4, 235-249.

26. Leiter, K.; Scheier, P.; Walder, G.; Märk, T. D. Int. J. Mass Spectrom. Ion Processes 1989, 87, 209-224.

27. Rademann, K.; Jochims, H. W.; Baumgärtel, H. J. Phys. Chem. 1985, 89, 3459-3464.

28. Leyh. B.; Wankenne, H. Int. J. Mass Spectrom. Ion Processes 1991, 107, 453-474.

29. Nguyen, M. T.; Kerins, M. C.; Hegarty, A. F.; Fitzpatrick, N. J. Chem. Phys. Lett. 1985, 117, 295-300.

30. Boyd, R. K.; Beynon, J. H. Org. Mass Spectrom. 1977, 12, 163-165.

31. Barber, M.; Elliott, R. M. 12th Annual Conference on Mass Spectrometry and Allied Topics; Montreal, 1964; ASTM Committee E14.

32. Todd, P. J.; McLafferty, F. W. Int. J. Mass Spectrom. Ion Phys. 1981, 38, 371-378.
33. Holmes, J. L. Org. Mass Spectrom. 1985, 20, 169-183.

34. Lias, S. G.; Bartmess, J. E.; Liebman, J. F.; Holmes, J. L.; Levin, R. D.; Mallard, W. G. J. Phys. Chem. Ref. Data 1988, 17, Suppl. 1.

35. Rosenstock, H. M.; Draxl, K.; Steiner, B. W.; Herron, J. T. J. Phys. Chem. Ref. Data 1977, 6, Suppl. 1.

36. Beynon, J. H.; Fontaine, A. E.; Lester, G. R. Int. I. Mass Spectrom. Ion Phys. 1972, 8, 341-363.

37. Holmes, J. L.; Osborne, A. D. Int. J. Mass Spectrom. Ion Phys. 1977, 23, 189-200.

38. Nakamura, H. Adv. Chem. Phys. 1992, 82, 243-319.

39. Landau, L. Phys. Z. Sowjetunion 1932, 1, 88-98.

40. Zener, C. Proc. Roy. Soc. London Ser. A 1932, 137, 696-702.

41. Demkov, Y. N. Sov. Phys. JETP 1964, 18, 138-142.

42. Rabalais, J. W. Principles of Ultraviolet Photoelectron Spectroscopy; Wiley: Chichester, 1977; p 92.

43. Egdell, R. G.; Fragala, I. L.; Orchard, A. F. J. Electron Spectrosc. Relat. Phenom. 1979, 17, 267-273.

44. Klots, C. E. Z. Naturforsch. 1972, 27a, 553-561.

45. Klots, C. E. J. Chem. Phys. 1973, 58, 5364-5367.

46. Klots, C. E. J. Chem. Phys. 1976, 64, 4269-4275.

47. Leyh-Nihant, B.; Lorquet, J. C.; McLafferty, F. W. Int. J. Mass Spectrom. Ion Processes 1990, 100, 465-475.

48. Manneback, C. Physica, 1951, 17, 1001-1010.

49. Herzberg, G. Molecular Spectra and Molecular Structure. II. Infrared and Raman Spectra of Polyatomic Molecules; Van Nostrand: New York, 1947; p 223.

50. Desouter-Lecomte, M.; Dehareng, D.; Leyh-Nihant, B.; Praet, M.-Th.; Lorquet, A. J.; Lorquet, J. C. J. Phys. Chem. 1985, 89, 214-222.

51. Köppel, H.; Domcke, W.; Cederbaum, L. S. Adv. Chem. Phys. 1984, 57, 59-246.

52. Shields, G. C.; Steiner, P. A., IV; Nelson, P. R.; Trauner, M. C.; Moran, T. F. Org. Mass Spectrom. 1987, 22, 64-69. 